\title{
PSYCHE
}

Vol. 70

JUNE, I963

No. 2

\section{THE VENOM AND POISON GLANDS OF PSEUDOMYRMEX PALLIDUS (F. SMITH)}

\section{By Murray S. Blum ${ }^{1}$ and Philip S. Callahan ${ }^{2}$}

In the United States, the small subfamily Pseudomyrmicinae is represented by several species which are known to sting severely. Probably the most common species in the southeastern area is Pseudomyrmex pallidus (F. Smith). As part of a program of study on ant venoms in our laboratories, this paper presents a characterization of the venom of this species, a description of its poison apparatus and a study of the reaction of human beings to its sting. In addition, the possible nature of the venoms produced by the Formicidae is discussed.

\section{The Poison Gland}

The poison gland of Pseudomyrmex pallidus is quite similar to that of Solenopsis saevissima, the imported fire ant, and the reader is referred to Callahan et al. (1959) for histological descriptions of the glands and sting of that species. As in the fire ant, the main gland of Pseudomyrmex pallidus is a rounded organ (Fig. ID) consisting of glandular collecting ducts which branch into lateral ducts that reduce in size and lead to the secretory cells. The round gland is inserted into an invagination of the tunica propria (E) which forms the poison sac. The stored poison of the sac empties through a main collecting duct $(\mathrm{F})$ into the base of the sting bulb $(\mathrm{H})$. There are two extremely long $(.49 \mathrm{~mm})$ free poison glands (A) which lead by way of a single neck (C) to the convoluted poison gland (D). The collecting ducts of the free glands (B) unite at the neck. In proportion to the rest of the organ, the neck is considerably longer $(.13 \mathrm{~mm})$ and the gland narrower than in the fire ant. Dufour's gland $(\mathrm{H})$ is extremely large in relation to the main poison gland, being $.4 \mathrm{~mm}$ in length or $4 / 5$ the length of the duct and sac of the

\footnotetext{
${ }^{1}$ Department of Entomology, Louisiana State University

${ }^{2}$ Department of Entomology, Louisiana State University; present address, Southern Grain Insects Laboratory, U.S.D.A., Tifton, Ga. Manuscript received by the editor August 10, 1962.
} 
main gland. In the fire ant it is only half the length of the duct and sac of the main poison gland.

\section{The Lancet}

The make-up of the lancet of Pseudomyrmex pallidus is also quite similar to that of the fire ant with two important exceptions. The first is that the valve (Fig. 2D) is inserted quite far back at the posterior junction of the lancet body $(G)$ with the ramus of the lancet $(\mathrm{C})$, the ramus being almost at right angles to the lancet. In
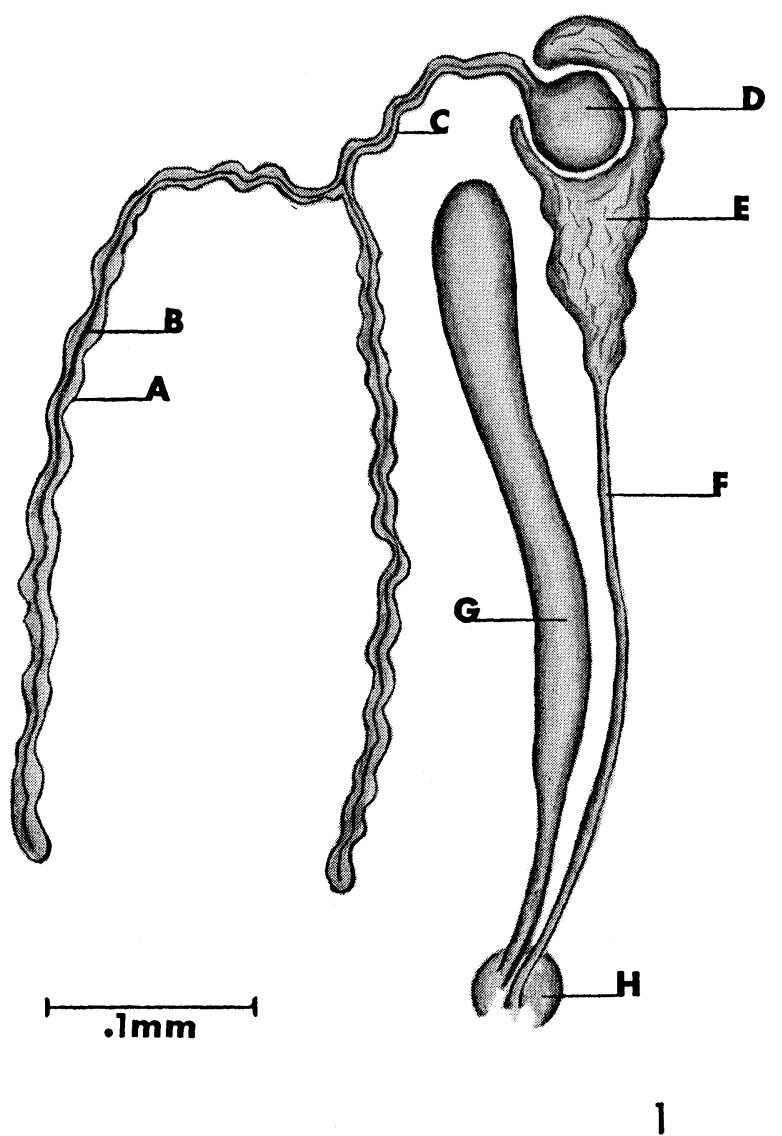

Figure 1. The poison glands of Pseudomyrmex pallidus. A, free poison gland; B, main collecting duct of free poison gland; C, neck of free poison gland; D, convoluted poison gland; E, tunica propria of poison sac; F, main duct of poison sac; G, Dufour's gland; H, base of sting bulb. 
the fire ant the valve is inserted forward on the lancet body (See Fig. 8. Callahan et al. 1959) and the ramus makes more of a curve where it joins the lancet. Secondly, unlike the fire ant, the tip of the lancet of Pseudomyrmex pallidus is not barbed.

\section{The Venom}

The venom of $P$. pallidus was obtained by holding the isolated abdomen of the ant by the petiole and collecting the venom issuing from the everted sting in microcapillaries or in the depression of a microscope slide. As many as twelve small droplets may be obtained by this method, after which the sting is invariably withdrawn, although the poison vesicle may still contain substantial quantities of venom.

The venom is a water-clear liquid which consists of a single phase. On contact with the air, each droplet solidifies into clear plates which can be easily chipped off the glass. However, if the venom is collected directly in fine capillaries, it retains its fluidity. Examination of the poison gland and Dufour's gland reveals that both contain waterclear liquids. The substance in the poison vesicle solidifies on contact with the air to form plates identical to those formed by the venom issuing from the sting.

The solubility of the venom was determined by allowing it to issue while the tip of the sting was held under different liquids. The venom is insoluble in distilled water and forms opaque strands which suspend in the aqueous medium. In organic solvents such as methanol, chloroform, $n$-hexane and diethyl ether, the venom forms insoluble, clear plates similar to those obtained when it is exposed to air. The venom is very soluble in dilute acids.

The venom is alkaline when tested with various indicator papers as an aqueous suspension. When the poison vesicle is crushed on indicator papers it also gives an alkaline reaction but interestingly, the viscous contents from crushed Dufour's gland are neutral. The secretions of the two glands are further contrasted by the fact that the fluid in Dufour's gland is soluble in organic solvents whereas the poison vesicle contents are not.

The venom of $P$. pallidus is ninhydrin-positive, producing a dark purple coloration with this reagent. Paper chromatographic analysis of the venom did not reveal the presence of any free amino acids; the ninhydrin-positive material all remained at the origin. All these data are consistent with the conclusion that the venom of this pseudomyrmicine ant is a basic protein(s). 
The small amount of venom available did not permit any further chemical characterizations.

The venom secreted from the sting of this ant is derived primarily, if not exclusively, from the true poison glands. Examinations of the poison glands of numerous ants from which venom had been collected frequently revealed that the poison vesicles were collapsed, whereas in every case Dufour's gland was distended with liquid. This was also the situation with glands from ants which had been allowed to sting human subjects. Although it is possible that traces of the secretion from Dufour's gland are secreted with the venom from the poison glands, it is quite evident that the biologically active secretion issuing from the sting of this ant essentially possesses the characteristics of the contents of the true poison glands.

\section{Skin Responses of Humans to the Sting}

Prior to stinging, the worker grips the skin with its mandibles and then arches the thorax upward so that the abdomen is bent underneath. The thorax and abdomen often form a sharply inverted $\mathrm{v}$ with a narrow angle between. This characteristic pose is a reflection of the elongate structure of these ants and probably provides the ant with sufficient leverage to drive the lancets into the skin. Although workers may sting for 30 seconds or more, multiple stings at one site were not frequently obtained.

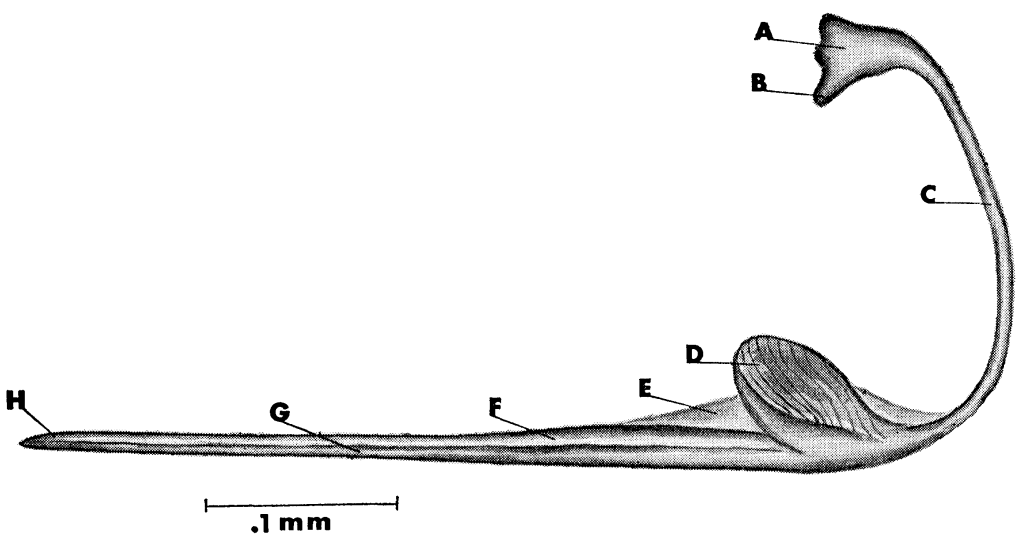

Figure 2. The lancet of Pseudomyrmex pallidus. A, triangular plate (first valvifer); $\mathrm{B}$, ventral a podeme of triangular plate; $\mathrm{C}$, ramus of lancet (first valvula) ; D, valve of lancet; $\mathrm{E}$, ligulate membrane; $\mathrm{F}$, dorsal fin of lancet; $\mathrm{G}$, main body of lancet; $\mathrm{H}$, tip of lancet. 
The skin response at the sting site is characterized by the immediate development of a flare, which reaches its maximum size in five to ten minutes. This is rapidly followed by the development of a wheal at the point where the lancets entered the skin. Within one hour the flare disappears and the area is marked only by the wheal which may persist for up to 24 hours. A dull pain is present after the ant has stung which becomes less intense within the first hour. A throbbing sensation is evident during this time which diminishes in intensity until by the end of the second hour very little discomfort is evident. Mild prurience is evident for at least 24 hours during which time a prominence develops at the site of the sting. This elevation is surrounded by a narrow red halo. After 48 hours, the puncture area is characteristically umbilicated and the depressed center is discolored, a condition which may persist for up to two weeks.

\section{Discussion}

One of the most significant characteristics about the venom of $P$. pallidus is that it is proteinaceous. Among the other stinging ants, only the myrmicine genus Solenopsis has been studied and it has been demonstrated that the venom is a non-protein (Blum et al. 1958; 1960). Thus, this pseudomyrmicine species is similar to vespid wasps and the honey bee in producing biologically active proteins in their poison glands whereas Solenopsis, a phylogenetically advanced myrmicine genus, differs in that its venom is non-proteinaceous.

The subfamily Pseudomyrmicinae is a relatively primitive one (Brown 1954) and it is tempting to speculate that proteinaceous venoms are characteristic of the more primitive subfamilies of ants. In support of this idea is the fact that the venoms of ponerine and doryline species that we have examined in our laboratories are proteinaceous. Along with the Pseudomyrmicinae, these families are more primitive than the Myrmicinae. In our laboratories, analyses of the venoms of the less highly developed myrmicines are now being carried out in order to determine whether or not this subfamily contains genera producing proteinaceous venoms as well.

It will of course be necessary to examine the venoms of many genera of ants in all subfamilies in order to determine whether or not proteins are limited to the phylogenetically less developed ones. It is well established that in the highly developed subfamily Formicinae, the poison glands produce the simple fatty acid formate, a considerable departure from the complexity of the proteinaceous or nitrogenous base-containing venoms found in Pseudomyrmex and Solenopsis respectively. 
The function of the Dufour's gland secretion in $P$. pallidus is completely unknown. This gland is highly developed in this ant (Fig. I) and contains at least as much material as the poison vesicle. The fact that this pseudomyrmicine species had a turgid Dufour's gland even after the poison vesicle had collapsed after secretion of the venom strongly indicates that the contents of Dufour's gland are secreted independently of the poison gland. In $P$. pallidus the $\mathrm{Du}-$ four's gland secretion is chemically very distinct from that of the poison gland. The fluid in Dufour's gland is a neutral non-protein which because of its high viscosity, is probably quite high boiling. In contrast, the poison gland contents consist of basic proteins which may not even be miscible with the secretion from Dufour's gland.

Wilson ( I959) has demonstrated that in the myrmicine Solenopsis saevissima Dufour's gland produces the trail laying substance in this species. Callahan et al. ( I959) demonstrated that the contents of Dufour's gland could be secreted independently of that of the poison glands. However, $P$. pallidus does not lay trails and indeed smears from Dufour's gland elicited no obvious responses from workers in a laboratory colony. Thus with the exception of the genus Solenopsis, the function of the Dufour's gland secretion in the Formicidae remains to be determined.

\section{References Cited}

Blum, M. S. and P. S. Callahan.

1960. Chemical and biographical properties of the venom of the imported fire ant (Solenopsis saevissima var. richteri Forel) and the isolation of the insecticidal component. XI Int. Kongr. Ent., Vienna. $3: 290-293$.

Blum, M. S., J. R. Walker, P. S. Callahan and A. F. Novak.

1958. Chemical, insecticidal, and antibiotic properties of fire ant venom. Science 128:306-307.

Brown, W. L.

1954. Remarks on the internal phylogeny and subfamily classification of the family Formicidae. Insects Soc. 1:22-31.

Callahan, P. S., M. S. Blum and J. R. Walker.

1959. Morphology and histology of the poison glands and sting of the imported fire ant (Solenopsis saevissima v. richteri Forel). Ann.

WILson, E. O. Ent. Soc. America 52:573-590.

1959. Source and possible nature of the odour trail of fire ants. Science $129: 643-644$. 

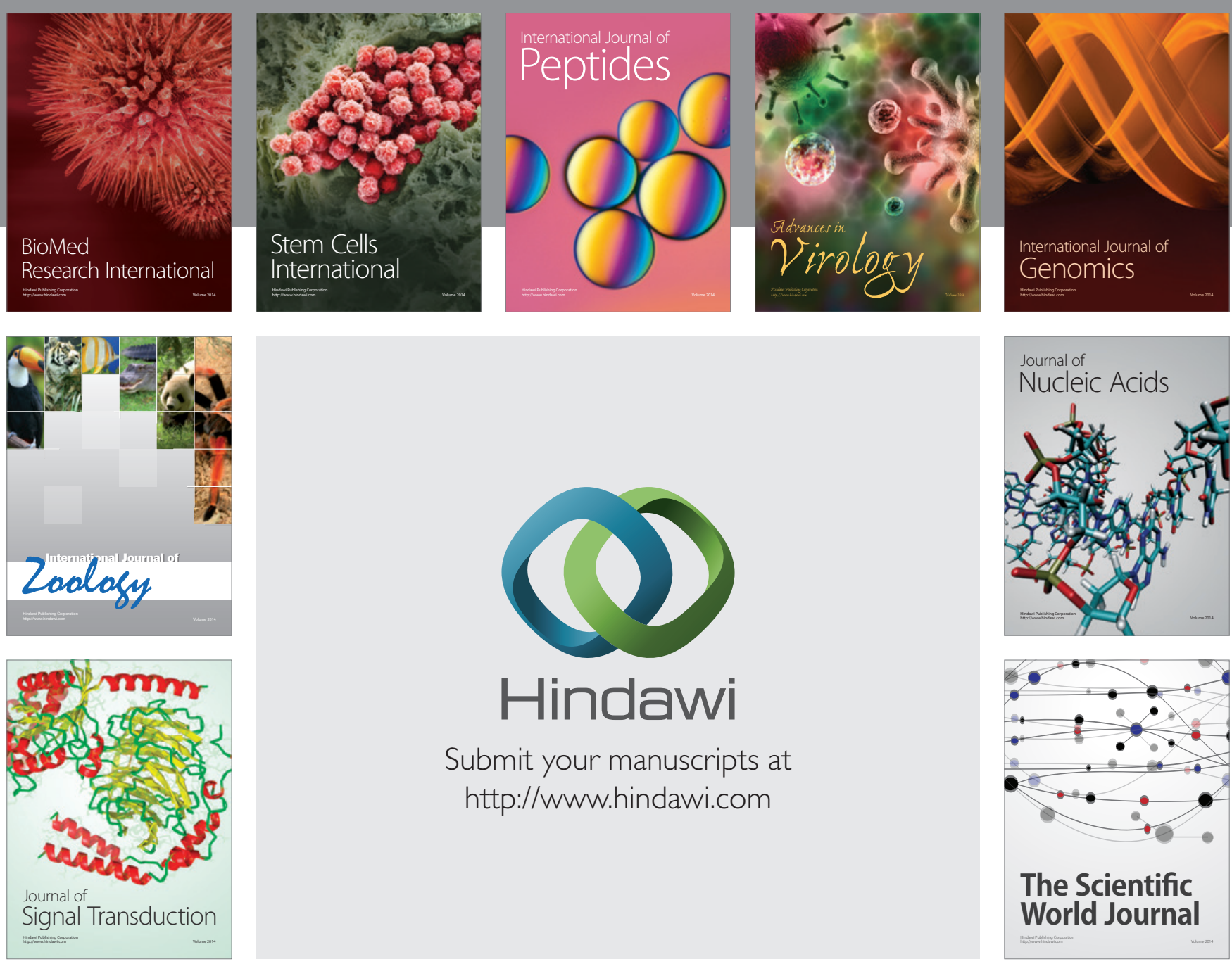

Submit your manuscripts at

http://www.hindawi.com
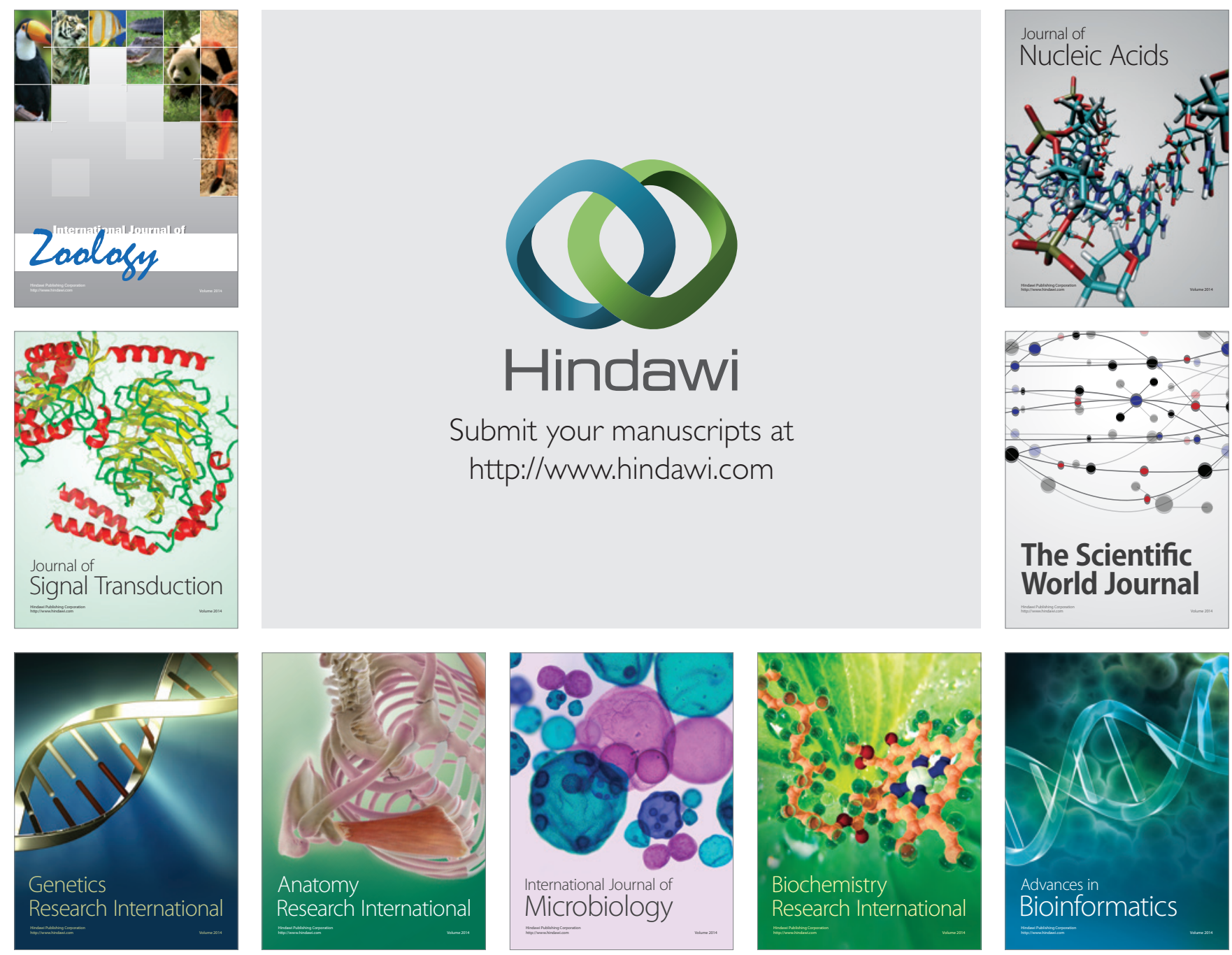

The Scientific World Journal
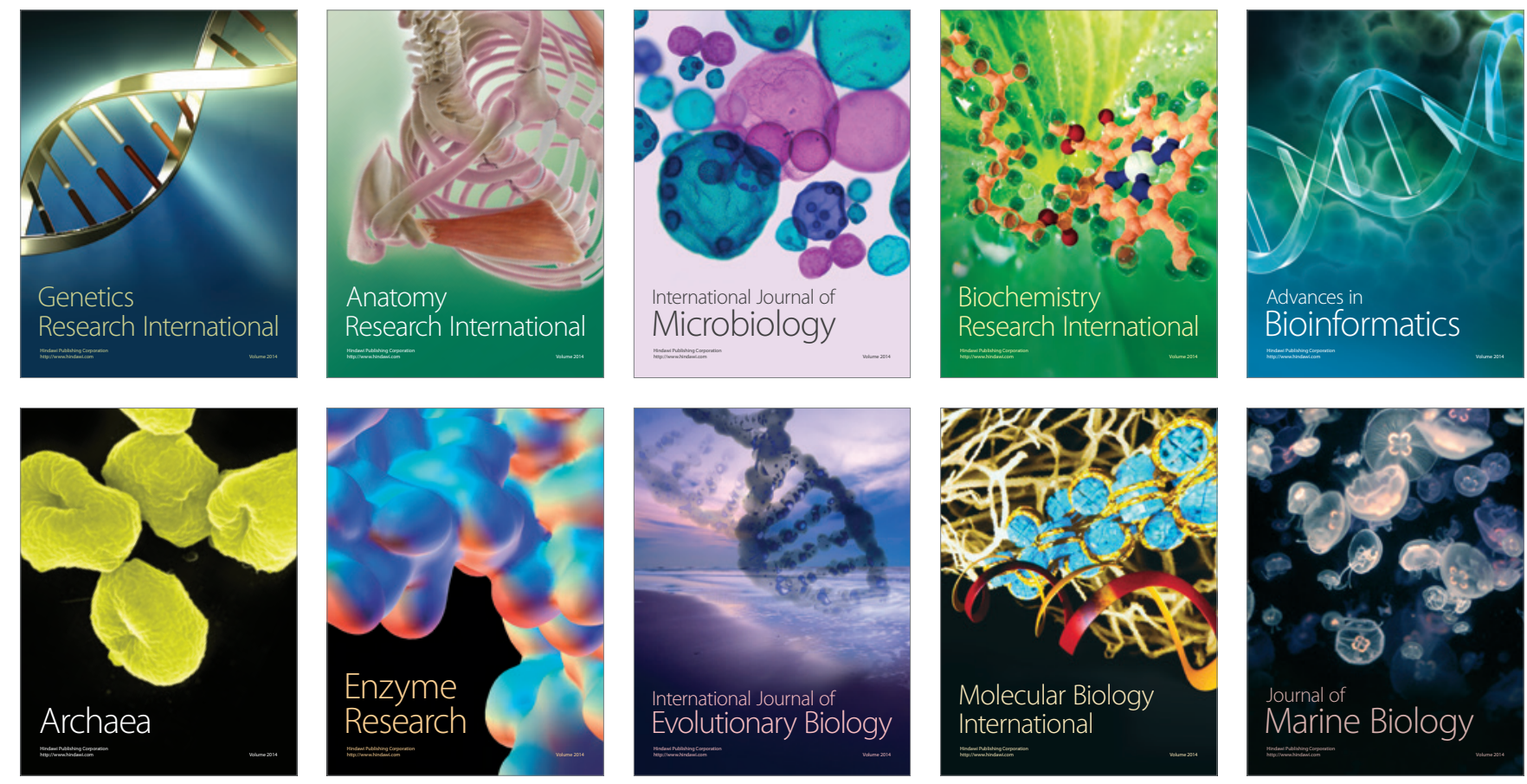